\title{
TURNING POINTS
}

\section{Finding CDK: Linking yeast with humans}

\section{Paul Nurse}

By the mid-1980s, I had been working on what controls the cell cycle in fission yeast for over a decade. My lab had identified the protein kinase $\mathrm{Cdc} 2$ as a cell cycle regulator that acts at both the G1-S and G2-M transitions, two major cell cycle control points. We had also shown that $\mathrm{Cdc} 2$ is equivalent to Cdc28, which Lee Hartwell had demonstrated as a key cell cycle regulator in budding yeast. However, whether Cdc2 or Cdc28 also had a role in metazoans remained unknown.

In 1984, when I moved to the Imperial Cancer Research Fund Institute in London, I quickly realised that if I were to convince my colleagues working on mammalian cells that yeast was more than just a nuisance that might infect their tissue cultures, I would need to find out whether or not humans also had Cdc2. Around this time, Melanie Lee joined my lab as a postdoctoral fellow. Among the projects we discussed, one was a 'safe' research option and another was to find the human Cdc2, should it exist. Typical of her character, Melanie chose the latter, a high-risk decision because at the time it was not at all obvious that controls of processes such as the cell cycle would be conserved from yeasts to humans, which had diverged during evolution approximately 1.5 billion years ago. In the 1980s, before wholesale genome sequences were available, the two conventional ways to find related genes were to look for similarities either at the protein level using antibodies, or at the level of the gene using DNA hybridization. As these approaches were labour intensive and time consuming, I assigned Martin Goss, the lab technician, to help Melanie. Martin rose to the occasion,

Paul Nurse is at The Royal Society, 6-9 Carlton House, London, SW1Y 5AG, UK.

e-mail: paul.nurse@royalsociety.org making the considerable workload bearable. Viesturs Simanis, a postdoctoral fellow skilled in molecular biology, was also very helpful in advising Melanie. But despite a lot of effort, neither approach worked. Occasionally, we would find a protein kinase that seemed promising. However, those results were difficult to interpret, because human cells were likely to have many hundreds of protein kinases. How would we know when we had the right one?

After nearly a year of work, we had a lab meeting to assess the situation. I have always used lab meetings to solve problems, because performing real discovery research is difficult and requires help from colleagues - which is why a supportive collegial atmosphere is crucial in a lab. During this particular lab meeting, we discussed the possibility of using functional equivalence rather than structural (DNA or protein) similarity. This was how we had shown that Cdc28 could substitute Cdc2 in yeast, as it complemented a temperaturesensitive $c d c 2\left(c d c 2^{t s}\right)$ mutant. However, there were potential problems with this approach: would functional equivalence still exist, given the 1.5-billion-year divergence, and were there human cDNA libraries available that could be expressed in fission yeast? Someone from the lab, either Tony Carr or Viesturs Simanis, suggested that a human cDNA library made by Hiroto Okayama in Paul Berg's lab might work. This library was driven by the SV40 early promoter which worked in fission yeast, so we could introduce it into yeast by co-transformation with a $c d c 2^{t s}$ mutant and look for complementation of the phenotype. A messy experiment, certainly - but it might just work. Hiroto and Paul gave us the library unhesitatingly, showing the generosity that makes the pursuit of science a pleasure. Melanie and Martin got to work, carrying out numerous transformations and optimization experiments, with Melanie relying on the much-appreciated support of my long-standing collaborator, Jacky Hayles, to keep going.

And then the experiment worked! Five colonies grew and the complementation seemed to be plasmid-borne, suggesting it was derived from the human library. Although the results were promising, the next couple of months were a nightmare, because we had to exclude other, less interesting but still possible, explanations. To be so close to such an exciting result, and yet to still fail, was almost unbearable. I would go home every evening telling myself, "I will imagine that it has worked, because when I go to the lab tomorrow, it will have failed - and I just want to savour the possibility of success for a short while!" The critical test was sequencing the gene that complemented the $c d c 2^{t s}$ mutant, but, at that time, sequencing was agonizingly slow. When the sequence was complete, it shared $61 \%$ identity with the fission yeast $c d c 2$ gene, but was of human origin. We had identified the human $\mathrm{Cdc} 2$, later named cyclin-dependent kinase 1 (CDK1). This experiment argued strongly that the cell cycle was controlled the same way in fission yeast and human cells, and therefore probably in all eukaryotic cells. This also meant that model organisms would be useful to study problems encountered in humans, even those relevant to diseases such as cancer.

This project worked because of Melanie's boldness, the support of her colleagues and the importance of the outcome, which provided the motivation to persist. Science is both intensely individual and completely collaborative. Often both are needed for success, and that was certainly the case in finding the human CDK. 